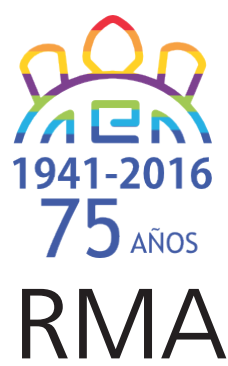

Arqueología

\title{
Organización espacial de la molienda en el sitio arqueológico Rincón Chico 1 (Catamarca, Argentina)
}

\author{
Spatial organization of the grinding at the Rincón \\ Chico 1 archaeological site (Catamarca, Argentina)
}

Natalia Silvana Petrucci*

*Museo Etnográfico "J. B. Ambrosetti", Facultad de Filosofía y Letras, Universidad Nacional de Buenos Aires. E- mail: nataliapetrucci@gmail.com

\begin{abstract}
Resumen
Rincón Chico 1 se ha definido como un poblado conglomerado, ubicado en el valle de Yocavil o Santa María. Si bien esta área ha sido objeto de múltiples investigaciones no se tenía un panorama completo de los instrumentos de molienda. El estudio de este tipo de artefacto en relación a su situación espacial radica no solo en que es posible contextualizarlos, sino también en que mostraría las posibles relaciones establecidas a partir de la organización del trabajo. Es por ello que el objetivo del presente trabajo es analizar la dinámica en torno a la molienda en el sitio arqueológico Rincón Chico 1, teniendo en cuenta las características morfológicas y la situación espacial o emplazamiento de los artefactos de molienda. Se tomaron medidas del diámetro de la boca y profundidad, así como las asociaciones de los morteros con estructuras arquitectónicas. En 5 sectores del sitio se registraron un total de 19 morteros (7 simples, tanto inmóviles como móviles) y 12 múltiples inmóviles, los cuales mostraron distintos tipos de asociación con estructuras arquitectónicas. Los resultados muestran que la organización grupal de la molienda prevalece a la individual, existiendo una sectorización marcada por la ubicación o el acceso de los artefactos.
\end{abstract}

Palabras clave: Valle de Yocavil; Rincón Chico 1; poblado conglomerado; dinámica de la molienda; situación espacial.

\begin{abstract}
Rincón Chico 1 has been defined as a cluster village, located in the Yocavil or Santa Maria valley. Despite the fact that this area has been the focus of several investigations, a complete picture of the grinding tools is missing. The study of this type of device in relation to its spatial situation lies not only in that it makes possible to contextualize them, but also in that it would show possible relationships established considering labor organization. That is why the aim of this paper is to analyze the dynamics associated with grinding at the Rincón Chico 1 archaeological site, taking into account the morphological features and the spatial location of the grinding tools. Measures of depth and diameter of the mouth were taken altogether with the associations between mortars and structures. In 5 sectors of the site a total of 19 mortars (7 simple, both stationary and mobile) and 12 multiple immobile, which showed different types of association with structures, were recorded. The results show that the communal organization of grinding prevails regarding to individual organization, and that there is a sectorization characterized by the location or the access to milling devices.
\end{abstract}

Keywords: Yocavil valley; Rincón Chico 1; cluster village; grinding dynamics; spatial location.

Los artefactos de molienda en sitios arqueológicos del Noroeste argentino (NOA), han sido estudiados a partir de su forma, tamaño, movilidad, funcionalidad, como fuente de información sobre la subsistencia de los grupos, aspectos vinculados a lo ceremonial o bien sólo se los ha mencionado como parte de los sitios. Desde hace varios años algunos autores han intentado resolver ciertos aspectos con respecto a estos instrumentos de molienda que no se habían abordado previamente, generando mayor interés en el estudio de este tipo de artefactos. Las depresiones, de origen antrópico, halladas en las rocas se han denominado de distintas maneras por distintos autores (Methfessel \& Methfessel 1998; Querejazu Lewis 1998). En Chile van Hoek (2003), en un intento de ordenar esta variedad de clasificaciones, propone diferenciar este tipo de depresiones en utilitarias y no utilitarias, llamándolas tacitas y cúpulas respectivamente. Otra diferenciación se plantea desde el punto de vista de la movilidad considerando, por una parte, artefactos móviles e individuales y, por otra, inmóviles y múltiples (los términos individuales y múltiples hacen referencia a la capacidad de que una o más personas puedan trabajar 
simultáneamente) (Giovannetti 2008; Giovannetti y González 2009). También se ha propuesto discriminar molinos de morteros a partir de la morfología y función. Los primeros poseen las oquedades de molienda dispuestas en sentido horizontal y tienen generalmente la forma de una batea (Giovannetti 2009). Los morteros se diferencian de los molinos porque la cavidad tiene un predominio vertical sobre el horizontal, suele ser profunda y de boca circular (Giovannetti 2009; Nardi y Chertudi 1969). En cuanto a las diferencias funcionales los molinos fueron destinados a la producción de harinas, en tanto que los morteros sirvieron para triturar y machacar granos. Babot (2004) plantea que ambos servirían para moler, pulverizar y triturar, mientras que los morteros también cumplirían la función de machacar y descascarar.

Estudios recientes se focalizaron en hallar posibles patrones en las formas de construcción y uso de los mismos (Giovannetti y González 2009). Babot (2007) ha definido una serie de referentes materiales que permiten distinguir casos de prácticas de molienda individual/ familiar o grupal así como el acceso o pertenencia de los artefactos. La importancia del estudio de este tipo de artefactos con relación a su situación espacial radica no solo en que es posible contextualizarlos, sino en que mostraría las posibles relaciones establecidas a partir de la organización del trabajo, quehaceres cotidianos o ciertas conductas relacionadas con la molienda.

Es por ello que el objetivo del presente trabajo es analizar la dinámica en torno a la molienda en el sitio arqueológico Rincón Chico 1, teniendo en cuenta las características morfológicas de los artefactos vinculados a dicha práctica, así como también la situación espacial o emplazamiento de los mismos a lo largo del sitio.

\section{El poblado Rincón Chico 1}

Rincón Chico 1 se ha definido como un poblado conglomerado, sobre un cerro escarpado y sus laderas, que pertenece a la localidad arqueológica de Rincón Chico, la cual se encuentra en el valle de Yocavil o Santa María, a pocos kilómetros de la ciudad de Santa María, Provincia de Catamarca. Hasta el momento se cuenta con 14 fechados para Rincón Chico 1 (sobre un total de 40 para la localidad) que muestran una ocupación más definida entre inicios del siglo XII y fines del siglo XV, es decir, en pleno momento de los Desarrollos Regionales (Tarragó 2011). El conjunto de este asentamiento residencial cubre una superficie de 40 hectáreas, combinando diversos grupos de estructuras de piedra distribuidos sobre la cumbre, el talud, la falda y el pie del cerro. A partir del registro de 365 estructuras arquitectónicas, se han definido 8 clases estructurales: plaza (espacio abierto delimitado por muro perimétrico), edificios redondos, recintos rectangulares comunicados, recintos asociados desiguales, plataformas (algunas decoradas con bloques blancos, negros y rosados); explanadas y muros de contención; hileras paralelas de lajas paradas (parapetos defensivos) y grandes bloques rocosos inmuebles con múltiples hoyos de molienda (Tarragó 1987, 1998). En función de la topografía y de las cuencas de escurrimiento, se efectuó originalmente una diferenciación interna de 12 sectores (Tarragó 1998), a los cuales se añadió el sector de los grandes peñascos con plataformas distribuidos hacia el este de las estructuras habitacionales, reconocido como sector XIII (González y Doro 2004; Reynoso 2003; Tarragó 2011). Los barrios residenciales y zonas públicas en la cumbre (sectores I a V); anfiteatro de erosión, que actúa como cuenca de escurrimiento estival del "arroyo de los Morteritos"; un área especial, la Quebrada del Puma, delimitada por dos espolones rocosos (Morro Norte y Morro Sur) con edificios de cuidada factura y muros decorados con bloques de colores, entre los que se encuentra una plataforma tricolor (sector VII); al E-SE del cerro se levantan cuatro segmentos constructivos que conforman el poblado bajo (sectores VIII, IX, X y XI) y un sector de edificios al sur de la torrentera meridional (sector XII). El sector XIII de grandes peñascos con orificios antrópicos (Tarragó 1998; 2011).

En estructuras residenciales del poblado bajo, sectores VIII, IX y X, se registraron los fechados más antiguos del poblado. Mientras que los valores relativamente más tardíos se observaron en sectores públicos como la cumbre y las adyacencias de la Quebrada del Puma (Greco 2012; Tarragó 2011).

\section{Evidencias de artefactos de molienda en Yocavil y áreas vecinas}

En los últimos años en el área se han realizado numerosos trabajos relacionados con este tipo de artefactos. En el sitio Rincón Chico 14 se definió un área de molienda integrada por cuatro morteros con cavidad circular, tres molinos planos, seis manos de molienda y una gran laja rectangular. Este contexto ha sido interpretado como un área de producción de alimentos a gran escala que superaría los requerimientos de un grupo domestico (Tarragó et al 1999).

Carbonelli (2009) contextualiza la producción de los artefactos de molienda y el rol que ocuparon en la vida cotidiana de las personas que habitaron el sitio Formativo o Temprano Soria 2 (Andalhuala-Banda). Siguiendo los indicadores que postula Babot (2007) concluye que la práctica de molienda se realizó de manera individual/ familiar y no grupal.

Los pocos artefactos de molienda hallados en superficie en el sitio de ocupación Temprano - Tardía Mesada del Agua Salada (Caspinchango) se encontraban partidos y formando parte de los muros. Las autoras interpretan, por una parte, que la poca cantidad de hallazgos puede deberse a que estos artefactos se encuentren enterrados $y$, por otra, que la ruptura pudo significar que las actividades 
de molienda dejaron de ser realizadas y se favoreció la construcción y mantenimiento de estructuras relacionadas a las actividades agrícolas (Álvarez Larraín y Lanzelotti 2013).

En el sitio El Cardonal es común la presencia de artefactos de molienda, los cuales poseen dos configuraciones espaciales bien delimitadas. Por un lado, los artefactos completos asociados espacialmente a manos de moler que sugieren la ocurrencia de actividades de molienda en dos recintos (E1 y E5) (Calo 2010). Por otro lado, artefactos de molienda rotos o desgastados o aquellos que son parte del material de construcción de la casa. En este último caso los autores distinguieron conanas o pecanas formando parte de los muros, o como jambas en las entradas de los recintos y manos de moler actuando como calzas o trabas (Scattolin et. al 2009).

Los artefactos de molienda inmóviles de Rincón Chico $1^{1}$ fueron registrados originalmente en planos detallados del sitio, con ubicación de GPS y como parte del inmueble del mismo. En el caso de los sectores VIII y IX, por la morfología de los artefactos y su vinculación a grupos de viviendas, se asumió que debieron estar destinados a la molienda de granos, marcando la sectorización de funciones (Tarragó 1998). Las excavaciones realizadas en el sector XIII demostraron que hubo actividad humana alrededor de éstos y los vestigios sugieren un uso ritual y funerario (González y Doro 2004; Tarragó 2011). Esta información junto con la registrada en la última campaña, nos permitirá tener un panorama completo en cuanto a la organización espacial en torno a la práctica de molienda en el sitio Rincón Chico 1.

\section{Metodología}

Para evitar confusiones con respecto a las clasificaciones que ya han sido dadas en Rincón Chico 1, hay que aclarar algunos términos que se emplearán en este trabajo. Se denominaran "megalitos" a los grandes peñascos naturales, que pueden o no poseer en su superficie orificios de molienda, "morteros" a la roca soporte (móvil o no) con la totalidad de sus orificios de molienda (sea uno o más) y "unidad de molienda" a los orificios individuales. Las expresiones simple y múltiple que utilizaremos tienen una connotación de cantidad, ya que los simples presentan un solo orificio de molienda y los múltiples más de uno. De esta manera evitamos confusiones cuando nos referimos al uso del artefacto y a la práctica de molienda en sí. Algunas rocas poseen pequeñas depresiones en su superficie producto de la erosión del agua, es por ello que siguiendo la clasificación de van Hoek (2003) para las "Tacitas" de Ovalle, se tuvieron en cuenta ciertas características a la hora de determinar los orificios de los

\footnotetext{
${ }^{1}$ El Lic. Jorge Sosa realizó el primer registro de las piedras de molienda de este sitio, a las que denominó "Tacanas", durante las campañas de levantamiento de estructuras, entre 1988 y 1994. La abreviatura "Tac." en los planos y tablas hace referencia a esta denominación.
}

morteros como unidades de molienda. Estas fueron: el diámetro entre $10 \mathrm{~cm}$ y $40 \mathrm{~cm}$, la forma de la boca entre circular y oval, el aspecto liso y la superficie horizontal o casi horizontal donde aparecen.

A partir de la definición de estos términos los morteros, inmóviles y móviles, así como todas sus unidades, fueron esquematizados a escala en el campo. De esta manera se pueden apreciar, no solo las dimensiones de estos artefactos, sino también el ordenamiento espacial entre estos y respecto a otras estructuras, al igual que de las unidades de molienda al interior de los mismos.

El conjunto de variables que se consideraron para el análisis y que se utilizaron para la descripción de los morteros hallados en el sitio Rincón Chico 1, fueron tomadas y adaptadas de Babot 2004, 2007, Giovannetti 2009. Las distintas categorías fueron:

Roca soporte: alude a la naturaleza geológica de la roca que constituye la piedra de molienda.

Movilidad: posibilidad de transportar la roca que contiene la unidad/es de molienda.

Característica cuantitativa: cantidad de oquedades, siendo simple si es una oquedad y múltiple si es más de una.

Agrupamiento: remitiría a dos o más unidades de molienda, encontradas en piedras de molienda múltiples, que pudieron fundirse en una compuesta.

Áreas bajo sedimento: si algún mortero se encuentra bajo sedimento (de manera total o parcial, en este último caso, considerando si el sedimento cubre o no un orifico de molienda), a fin de evaluar la posibilidad de hacer una extracción de sedimento para realizar análisis de microrrestos que fuera susceptible de reflejar sus usos en el pasado.

Ubicación en el paisaje: esta categoría tiene en cuenta la dinámica del ambiente (por ejemplo, cárcavas) y relación con recintos $u$ otras estructuras.

Otras variables que se tuvieron en cuenta para el relevamiento de las unidades de molienda fueron:

-Diámetro mayor de la boca

-Diámetro menor de la boca

-Profundidad

-Bordes pulidos (presencia/ausencia)

Los dos diámetros de la boca que se tienen en cuenta permiten conocer la morfología de los orificios en planta, permitiendo contar con un esquema a escala de esta vista. Estos datos, sumados a la profundidad, permiten el esquema de perfil de las unidades de molienda. La presencia de bordes pulidos es un indicador del uso de los orificios, lo cual genera el pulimento.

A partir de los datos registrados, se confeccionó una tabla con los tipos de asociación de cada mortero con estructuras arquitectónicas. Las categorías fueron: 


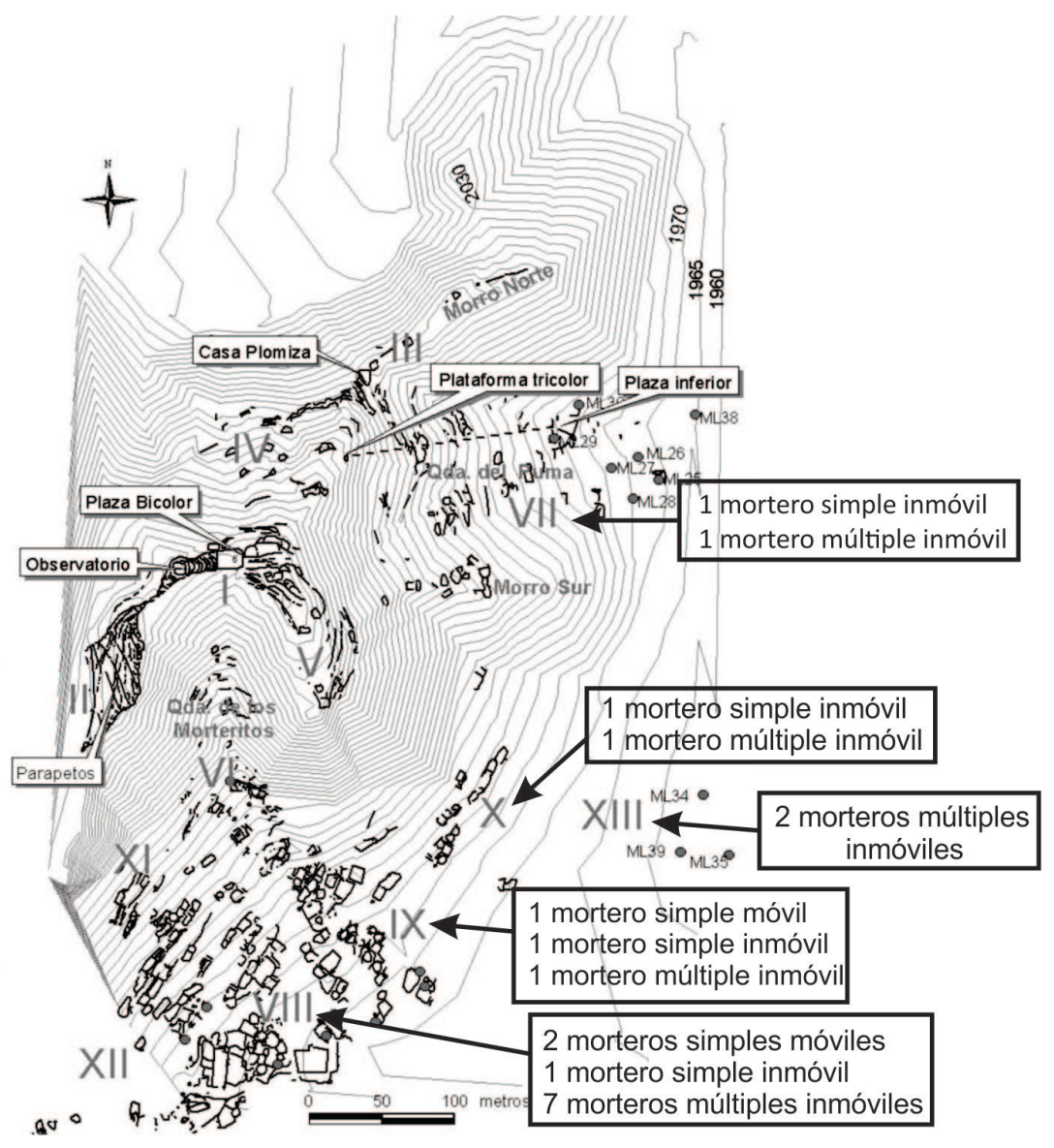

Figura 1: Mapa de Rincón Chico 1 con ubicación, tipo y cantidad de morteros, hallados en superficie, en cada sector (modificado de Tarragó 2011).

Figure 1: Map of Rincón Chico 1 with location, type and amount of mortar, found on the surface in each sector (modified from Tarrago 2011).

En el sector IX un megalito posee una única unidad de molienda, habiendo podido albergar más, considerando sus dimensiones $(6 \times 5,2 \mathrm{~m})$. Éste ocupa casi la totalidad de una estructura parcialmente delimitada por paredes poco definidas, que se trataría de una explanada tipo patio de trabajo (com. per. Tarragó 2014) (Figura 3).

La tabla 2 muestra las dimensiones de las unidades de molienda, donde se puede observar que los valores del diámetro varían entre 10 y $25 \mathrm{~cm}$. Salvo en un caso, las medidas del diámetro de la base, coincidían con el diámetro de la boca. Se pudieron determinar dos tipos morfológicos de boca: circular (cuando todos los puntos equidistan al centro) y semicircular a) cerca o próximo a recintos, b) dentro de recintos, c) formando parte de los muros y d) no asociada a estructuras arquitectónicas.

\section{Resultados}

En total se relevaron 19 morteros en superficie, ubicados en el piedemonte del cerro, de los cuales 7 son simples (inmóviles y móviles) y los 12 restantes son múltiples (inmóviles). Los megalitos que poseen unidades de molienda tienen dimensiones que varían entre 3 y $24 \mathrm{~m}^{2}$ aproximadamente. De los 13 sectores en que se dividió el sitio, a partir de la topografía y de las cuencas de escurrimiento (Reynoso 2003; Tarragó 2011), sólo en 5 se encontraron artefactos de molienda simples (inmóviles y móviles) como múltiples (inmóviles) (Figura 1). El sector VIII presenta la mayor cantidad de morteros.

La tabla 1 muestra la distribución de los morteros hallados en superficie en el sitio y la asociación o no con estructuras arquitectónicas. En el sector VIII, 6 megalitos de naturaleza pegmatítica forman parte de los muros de estructuras que se definieron, a partir del plano y de la observación en campo, como posibles "patios", es decir recintos grandes, de paredes bajas y no techados (com. pers. Tarragó 2014). En dos de los cuales hay unidades de molienda en su superficie (Figura 2). A partir de las excavaciones se pudo determinar que los recintos, próximos a estos "patios", eran de carácter residencial. (cuando uno de los ejes es más elongado que el otro, no llegando al extremo de ser elipsoidales). En corte longitudinal, los orificios de molienda exhibieron una morfología cilíndrica o subcilíndrica, de paredes verticales y fondo levemente curvo y con un diámetro similar al de la boca. Las profundidades son variables dentro de cada mortero. Por ello se calcularon las profundidades promedios para los poblados y sectores ceremoniales en conjunto, constatándose que los mayores valores de profundidades promedios se observaron en los poblados (sectores VIII, IX y X).

Se decidió no hacer extracción de sedimentos para realizar análisis de microrrestos, ya que todos los morteros se encontraban expuestos. Se observaron contaminaciones actuales en las unidades de molienda tanto antrópicas (fogón dentro de las unidades de molienda) como de animales (guano, orina y semillas de Prosopis sp).

\section{Discusión}

Analizando la organización espacial de los distintos morteros relevados en superficie nos encontramos con situaciones similares en otros sitios del NOA, así como disposiciones aún no registradas o documentadas. De los morteros registrados como simples móviles, que implican una práctica de molienda individual (o a lo sumo de dos personas) definida por la restricción impuesta por una única oquedad (Babot 2007), encontramos dos 


\begin{tabular}{|c|c|c|c|c|c|}
\hline Sector & $\begin{array}{l}\text { Mortero/ } \\
\text { Ubicación }\end{array}$ & $\begin{array}{c}\text { Cerca o próximo } \\
\text { a recintos }\end{array}$ & $\begin{array}{l}\text { Dentro de } \\
\text { recintos }\end{array}$ & $\begin{array}{l}\text { Formando parte de } \\
\text { los muros }\end{array}$ & $\begin{array}{c}\text { No asociado a } \\
\text { estructuras } \\
\text { arquitectónicas }\end{array}$ \\
\hline \multirow{10}{*}{ VIII } & $\begin{array}{l}\text { Tac Qda De } \\
\text { Morteritos I }\end{array}$ & $x$ & - & - & r \\
\hline & $\begin{array}{l}\text { Tac Qda de los } \\
\text { Morteritos II }\end{array}$ & $x$ & - & - & - \\
\hline & Tac ML2 & $x$ & - & - & - \\
\hline & Tac ML5 & - & - & $x$ & - \\
\hline & Tac ML6 & - & - & $X$ & - \\
\hline & Tac ML7 & $x$ & - & - & - \\
\hline & Tac ML9 & $x$ & - & - & - \\
\hline & Tac ML10 & $x$ & - & - & - \\
\hline & Tac R138* & - & - & $x$ & - \\
\hline & Tac R148* & - & $x$ & - & - \\
\hline \multirow{3}{*}{ IX } & Tac R163* & - & - & $x$ & - \\
\hline & Tac R166 & - & $x$ & - & - \\
\hline & Tac R193 & $x$ & - & - & - \\
\hline \multirow{2}{*}{$X$} & Tac SX & - & - & - & $X$ \\
\hline & Tac ML40 & - & - & - & $x$ \\
\hline \multirow{2}{*}{ VII } & Tac ML26 & - & - & - & $X$ \\
\hline & Tac ML28 & - & - & - & $x$ \\
\hline \multirow{2}{*}{ XIII } & Tac ML34 & - & - & - & $X$ \\
\hline & Tac ML35 & - & - & - & $x$ \\
\hline
\end{tabular}

Tabla 1: Tipos de asociación de morteros con estructuras en el sitio Rincón Chico 1. Referencia: * mortero móvil.

Table 1: Types of mortars association structures on the site Rincón Chico 1 Reference: * Mobile mortar.

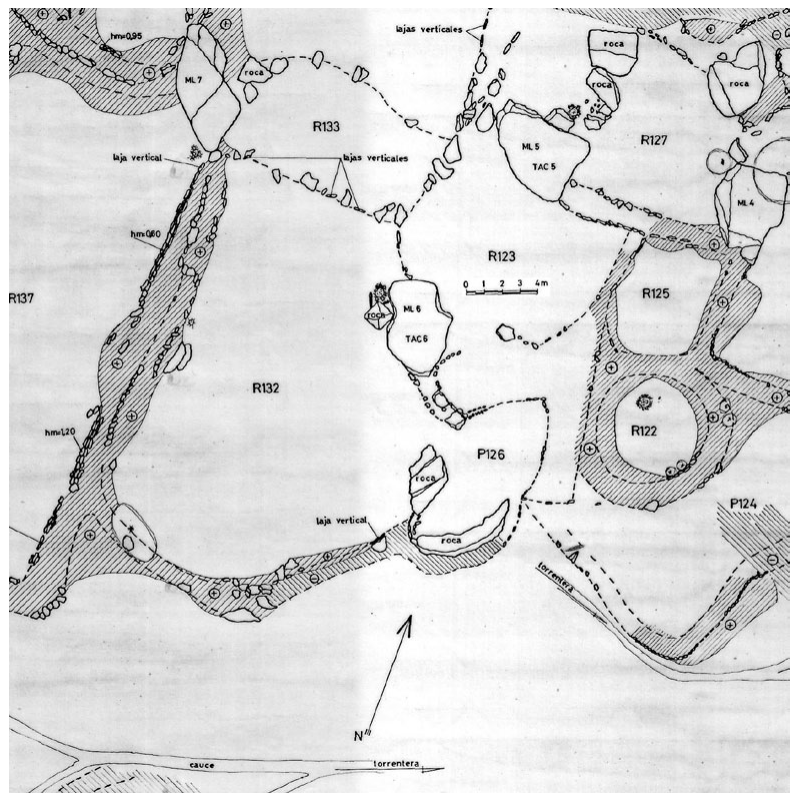

Figura 2: Plano Sector VIII a escala mostrando los megalitos que poseen unidades de molienda y forman parte de los muros. Referencias: ML: megalito; P: plataforma; Tac: tacana; R: recinto.

Figure 2: Map showing scale Sector VIII megaliths possessing grinding units and part of the walls. References: ML: megalith; P: platform; Tac: Tacana; A: exhibition.

situaciones bien diferenciadas. Por un lado dentro del recinto 148, sector VIII, que conforma un espacio cerrado, se encontró un mortero en posición de uso. El mismo poseía una fractura que se podría pensar que sucedió posterior al abandono del sitio, ya que en otros casos registrados hay evidencias de que estos artefactos tras su rotura, eran reciclados como rocas de muro (Álvarez Larraín y Lanzelotti 2013; Babot 2007, Scattolin et. al

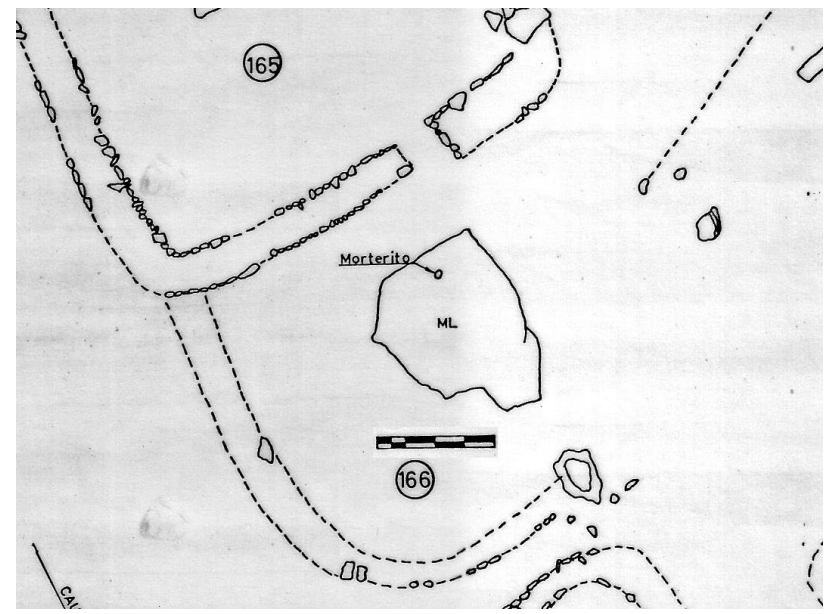

Figura 3: Plano mostrando el mortero simple que ocupa casi la totalidad de la estructura $n^{\circ} 166$, del sector IX, del sitio Rincón Chico 1 (escala = 4 metros). Referencias: ML: megalito; 165 y 166: recintos.

Figure 3: Map showing the simple mortar occupies almost the entire structure No. 166, Sector IX, Site Rincón Chico 1 (scale = 4 meters). References: ML: megalith; 165 y 166: structure.

2009). Se hallaron también dos morteros fracturados, en el sector VIII y IX, como roca de muro, por lo cual se puede pensar que hubo un reciclado de los mismos como material de construcción, al igual que registran otros autores (Álvarez Larraín et al 2013; Babot 2007 Scattolin et. al 2009) para numerosos casos del NOA. Poco puede decirse a partir de estos restos sobre las actividades de molienda y accesibilidad a los mismos, ya que su inclusión en el muro fuera de su contexto de uso para molienda, nos impide sacar más conclusiones sobre dicha práctica. La poca cantidad de este tipo de 


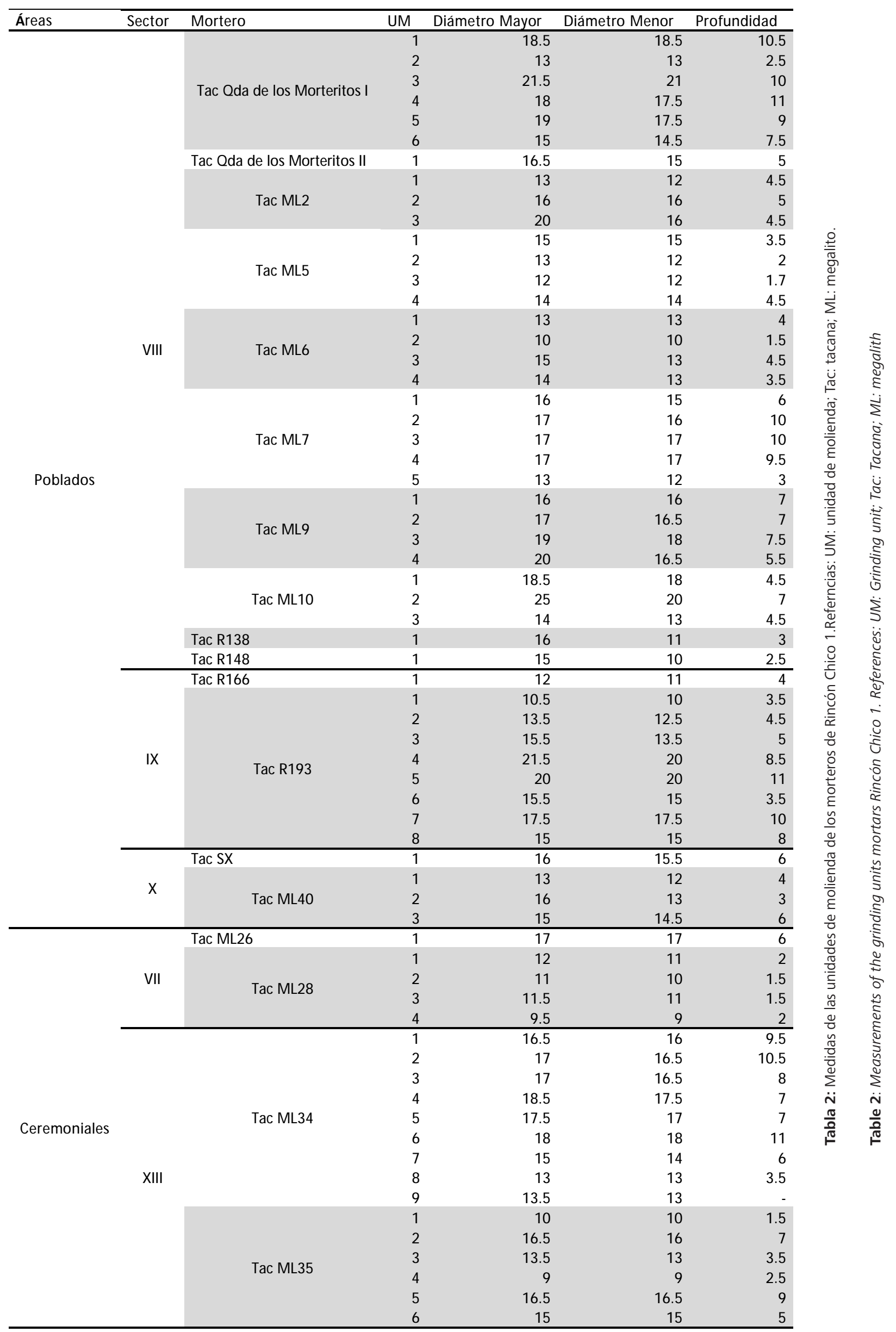


artefactos en comparación con los morteros simples y múltiples inmóviles, puede deberse a que los mismos se encuentren enterrados, como lo registrado en el sitio Rincón Chico 14, de la misma localidad arqueológica (Tarragó et al. 1998).

En el sector $X$, encontramos un mortero simple inmóvil, en un espacio abierto cerca de una cárcava y no asociado a estructuras arquitectónicas. Al no estar delimitado por estructuras, se sugiere que el acceso no era restringido a las distintas unidades familiares que pudieron habitar el sitio.

Otro caso lo conforman dos megalitos, que se definieron como morteros simples por poseer una única oquedad, pero que por sus dimensiones tienen la potencialidad de albergar más de un orificio. Uno de ellos, ya mencionado, se encuentra en el interior de la estructura 166 del sector IX, ocupando casi la totalidad de este (Fig. 3), de manera que el acceso a dicho instrumento pudo estar limitado a las unidades familiares que integraban los recintos contiguos. El otro megalito se ubica en el sector VIII, cerca del anterior, en un espacio abierto, no delimitado por estructuras, próximo a un mortero múltiple. En este caso tanto el mortero simple como el múltiple conformarían los artefactos de molienda de este espacio cercano a los recintos de los sectores VIII y IX, pudiendo indicar que la actividad llegó a realizarse en forma simultánea por las unidades familiares que habitaban los recintos cercanos. Así como el cuidado pudo realizarse también entre varias unidades domésticas.

Además se registraron grandes morteros con numerosas oquedades, que pueden implicar una práctica de molienda individual o grupal, en lugares abiertos que se localizan cerca de los márgenes o accesos a los recintos (en el sector VIII Tac ML3, Tac ML7, Tac ML9 y Tac ML 10 y en el sector IX Tac R193), como sucede en otros sitios del NOA (sitios Punta de la Peña 9, Punta de la Peña 4, Punta de la Peña 4-pie de talud y El Sembrado-alero, en Antofagasta de la Sierra) (Babot 2007). Siguiendo a Babot (2007), podemos pensar que aunque estos espacios no están delimitados por estructuras, la proximidad a los recintos, algunos adosados a estos, podría indicar una organización grupal, de pertenencia y de acceso libre para los grupos familiares que habitaban los recintos contiguos.

Dentro de los morteros con múltiples oquedades encontramos un megalito en el sector $X$, con 4 orificios (mortero Tac ML 40) no asociado a estructuras arquitectónicas que puedan sugerir un acceso restringido al mismo por parte de las distintas unidades familiares que habitaban el sitio. Por el contrario, en el sector VIII se pudo registrar que grandes peñascos de pegmatita formaban parte de los muros, dos de los cuales además cumplieron una doble función, ya que en su superficie poseían oquedades de molienda (Tac ML5 y Tac ML6) que se asume fueron accesibles durante el momento de funcionamiento del sitio. Esto podría indicar una restricción al acceso y pertenencia de estos artefactos, implicando posiblemente cierta especificidad en la molienda, donde los grupos familiares de los recintos próximos, definidos como residenciales, eran las que pudieron de cierto modo regular el uso de dicho espacio. Es imposible pensar que estos grandes peñascos hayan sido transportados a un lugar donde se proyectaba realizar una edificación, sino que hubo un aprovechamiento del lugar donde quedaron estas grandes rocas, luego de desprenderse naturalmente del tronco montañoso, para emplazar las distintas estructuras. De esta manera los megalitos con y sin orificios de molienda no solo estarían estructurando el espacio, sino que también estarían articulando las distintas estructuras que conformaban unidades residenciales de una misma unidad doméstica -articulando una o más familias- que compartían el acceso a estos morteros de gran tamaño. Esta es una situación que solo se da en este sector, el poblado bajo, siendo de esta manera un carácter que lo distingue de los otros. A partir de lo anterior y de los fechados obtenidos también se puede pensar que este sector fue el núcleo original del sitio (com. pers. Tarragó 2014), ya que es de los más antiguos registrados en el sitio (Greco 2012; Tarragó 2011).

Por último nos quedan los megalitos ubicados en los sectores VII y XIII, que son grandes peñascos rodeados por plataformas pircadas. En ambos casos las excavaciones realizadas permitieron asignarles un carácter ceremonial, ya que las evidencias recuperadas indican que en estos lugares se efectuaron enterratorios humanos y, tal vez en el sector VII sacrificios de camélidos. En el sector XIII también se halló un fragmento de campana oval de bronce (González y Doro 2004). Algunos de estos megalitos poseen orificios de molienda en su superficie, pudiendo indicar que la práctica de molienda en estos sectores estaría vinculada con ciertos rituales y el acceso restringido a esos momentos y a las personas responsables de efectuarlos.

Es conocido, como ya lo han mencionado otros autores (Fernánadez Distel 1994; Giovannetti y González 2009; Nardi y Chertudi 1969-70; Rusconi 1945; Serrano 1945), que la variabilidad en las profundidades, sea dentro de cada mortero múltiple como entre los mismos, se deba a la especificidad funcional de cada unidad de molienda, a la intensidad y frecuencia de uso, o bien a la antigüedad de los artefactos. En Rincón Chico 1 las diferencias entre las unidades de molienda en un mismo mortero se podrían entender por medio del mismo fenómeno, con unidades que pudieron ser más intensamente usadas que otras o elaboradas en distintos momentos. Para la variabilidad entre los distintos morteros podemos plantear lo mismo, ya sea por la intensidad de uso o por la antigüedad de los mismos. Las mayores profundidades promedio coinciden con el componente más antiguo del 
sitio, que se encuentra en estructuras, definidas como residenciales, del poblado bajo (sectores VIII, IX y X) (Greco 2012; Tarragó 2011). Ocurre lo contrario cuando analizamos la morfología de la boca, donde encontramos uniformidad, entre las unidades de molienda de un mismo mortero como entre ellos, siendo estas circulares o semicirculares. Giovannetti y González (2009) plantean para el sitio El Shinkal de Quimivil que la homogeneidad entre los morteros, teniendo en cuenta solo la morfología, apoyaría la hipótesis de que estos artefactos pudieron ser construidos (de manera estandarizada) y utilizados en un mismo momento y para las mismas finalidades funcionales.

Como expresábamos anteriormente, los términos en que nos referimos a morteros simples y múltiples, es una clasificación que solo tiene en cuenta la cantidad de oquedades presentes, de esta manera evitamos confusiones cuando nos referimos a la práctica de molienda (individual y grupal). Babot (2007) plantea que no es suficiente el conteo de oquedades para darle un carácter grupal a la práctica de molienda y propone un conjunto de variables morfológicas, como las dimensiones de las oquedades, sus superficies activas y la proximidad entre ellas. Tomando en cuenta esas variables calculamos un número máximo de operarios que pudieron trabajar simultáneamente sobre los morteros múltiples. En Rincón Chico 1 encontramos que el Tac ML 40 con tres oquedades próximas, con cierta continuidad entre ellas, establecida por el desgaste, y con dimensiones diferentes, pudo ser utilizado por un solo operario -o a lo sumo doscon distintos propósitos. Por otro lado establecemos que tres o más oquedades pudieron ser utilizadas por más de dos operarios al mismo tiempo, en este sentido se podría decir que la práctica de molienda pudo realizarse de manera grupal. Este es el caso de los morteros Tac ML 5, el Tac ML 10 Tac ML 6, Tac ML 9, Tac ML 7 y el Tac ML 3 (Sector VIII) Tac Quebrada I (sector VI) y Tac R 193 (sector IX) (Figura 4).

\section{Conclusiones}

Existen varios puntos que se pueden destacar a partir del análisis realizado. Por un lado, la ubicación restringida de estos artefactos en los sectores del poblado bajo y en espacios determinados como ceremoniales. El poblado bajo (Sectores VIII, IX y X) constituye un espacio donde la molienda fue una práctica importante, sobre todo en el sector VIII, por la gran abundancia de estos artefactos, donde la accesibilidad a estos instrumentos se puede pensar que estuvo mayormente restringida, o en todo caso pautada, por los habitantes de los recintos próximos o contiguos. Por otro lado, en términos generales podemos decir que la organización grupal en torno a la molienda prevalece a la práctica individual.

Se pudo establecer que grandes peñascos (con y sin unidades de molienda en su superficie) estructuraban y

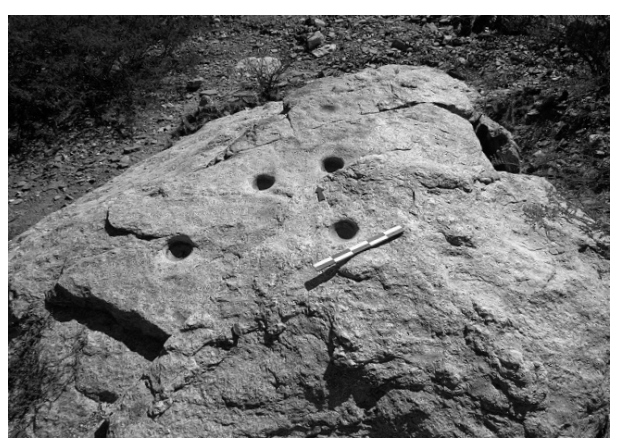

Figura 4: Mortero Tac ML 7, sector VIII.

Figure 4: Mortar Tac ML 7 Sector VIII.

articulaban el espacio doméstico en el sector VIII, siendo un carácter que lo diferencia en cuanto a lo constructivo y a la organización de la molienda.

Rincón Chico 1 sería uno de los primeros sitios donde se registra la presencia de morteros múltiples fijos como parte de los muros de estructuras establecidas como "patios", los cuales comparten sus muros con las unidades residenciales próximas. Estos morteros están cumpliendo un rol fundacional en la construcción, antes que como producto de un proceso de reciclado tras quedar inutilizados para la molienda (como el caso de morteros móviles). Los morteros múltiples de gran tamaño actuaron, en sectores específicos del sitio, como elementos fundadores, estructurantes y articuladores del espacio doméstico y posiblemente permanecieron activos tras ser materialmente apropiados a través de los muros que los fueron ciñendo al quehacer doméstico de las familias de este poblado del tardío en Catamarca. Esto apoyaría la hipótesis, junto con los fechados y los datos de las profundidades promedios, de que estos sectores del poblado bajo fueron el núcleo original del sitio, sobre todo el sector VIII.

A modo de cierre podemos decir que existía una sectorización en torno a la molienda, marcada por la ubicación o el acceso de los artefactos, siendo el poblado bajo el lugar donde se realizaban dichas actividades presumiblemente de modo cotidiano y los sectores VII y XIII en momentos vinculados al ritual.

La Plata, 1 de septiembre 2014

\section{Agradecimientos}

Expreso mi agradecimiento a la Dra. Myriam Tarragó que generosamente me dio acceso al material, resultado de tantos años de trabajo. A la Dra. Verónica Lema que siempre me acompaña, guía y entusiasma a seguir. Al Lic. Jorge Sosa por el material aportado. Al Dr. Catriel Greco que en sucesivas campañas me enseño, explico y alentó con el trabajo. A las excelentes compañeras de campo, Lic. Leticia Raffaele y a Silvina Aumont por ayudarme a relevar los morteros. Al Dr. Juan Pablo Carbonelli por la 
revisión del trabajo y aporte de ideas. A la Dra. Sonia Lanzelotti por su ayuda con los planos y contribuir con bibliografía. Y a todo el equipo de Yocavil que siempre están bien predispuestos a colaborar, escuchar y construir.

\section{Bibliografía}

Álvarez Larraín, A. y Lanzelotti, S. 2013. Habitar y cultivar en el este del valle de Yocavil. Editado por Inés Gordillo y José María Vaquer, La espacialidad en arqueología. Enfoques, métodos y aplicación, pp. 151-190. Editorial Abya-Yala, Quito, Ecuador.

Babot, M. 2004 "Tecnología y utilización de artefactos de molienda en el Noroeste prehispánico". Facultad de Ciencias Naturales e IML, Universidad Nacional de Tucumán, Argentina, 303 pp. Tesis Doctoral, San Miguel de Tucumán.

Babot, M. 2007. Organización social de la práctica de la molienda: casos actuales y prehispánicos del Noroeste Argentino. Compilado por A. Nielsen, M. C Rivolta, V. Seldes, M.M. Vázquez y P. Mercolli, Procesos sociales prehispánicos en el sur andino. La vivienda, la comunidad y el territorio; pp. 259-291. I Brujas, Argentina.

Calo, C. M. 2010. Plantas útiles y prácticas cotidianas entre los aldeanos al sur de los Valles Calchaquíes (600 A.C.-900 D.C.). Tesis Doctoral. Facultad de Ciencias Naturales y Museo, Universidad de La Plata, La Plata

Carbonelli, J. P. 2009. "Interacciones cotidianas entre materias primas y sujetos sociales en el Valle de Yocavil. El caso del sitio Soria 2 (Andalhuala, Pcia. de Catamarca)". Facultad de Filosofía y Letras, Universidad de Buenos Aires, Argentina, 144 pp. Tesis inédita de licenciatura en Ciencias Antropológicas (orientación en Arqueología), Facultad de Filosofía y Letras.

Fernández Distel, A. 1994 Noticias sobre el sitio arqueológico de Abra de Los Morteros y otros lugares de valor prehistórico en la región de Santa Bárbara (Jujuy, República Argentina). Editado por Albeck M. De Costa a Selva. Producción e intercambio entre los pueblos agroalfareros de Los Andes Centro Sur. Pp: 255-294 Instituto Interdisciplinario de Tilcara, FFyL, UBA.

Giovannetti, M. 2008 Los morteros múltiples en el Noroeste Argentino: un enfoque interregional. Compilado por Austral, A. y Tamagnini, M. Problemáticas de la arqueología contemporánea. Tomo III. Pp. 773-782, Universidad Nacional de Rio Cuarto, Argentina.

Giovannetti, M. 2009 "Articulación entre el sistema agrícola, redes de irrigación y áreas de molienda como medida del grado de ocupación Inka en El Shincal y Los Colorados (Prov. de Catamarca)". Facultad de Ciencias Naturales y Museo, Universidad Nacional de La Plata, Argentina, 794 pp. Tesis de doctorado inédita.
Giovanetti, M. y P. González 2009 Análisis de la variación métrica en morteros múltiples de El Shincal de Qumivil, Catamarca. Editado por Palacios O. M., Vázquez C., Palacios T. y Cabanillas E. Arqueometría latinoamericana, volumen 1, pp. 374-380. Comisión Nacional de Energía Atómica, Centro Atómico Constituyentes, Buenos Aires.

González, L. R. y R. Doro. 2004. Jardines de piedra. Estructuras ceremoniales en Rincón Chico. Etnia 4647:147-168. Olavarría.

Greco, C. 2012 "Integración de datos arqueológicos y geofísicos para la construcción de una cronología absoluta de Yocavil y alrededores". Facultad de Filosofía y Letras, Universidad de Buenos Aires, Argentina, 497 pp. Tesis para optar al grado de Doctor en Ciencias Antropológicas (orientación en Arqueología), Facultad de Filosofía y Letras, Universidad de Buenos Aires. Manuscrito.

Korstanje, M.A. 2005. “La Organización del Trabajo en Torno a la Producción de Alimentos en Sociedades Agropastoriles Formativas (Pcia. de Catamarca, Rep. Argentina)". Facultad de Ciencias Naturales e Instituto Miguel Lillo, Universidad Nacional de Tucumán, Argentina, 405 pp. Tesis de Doctorado en Arqueología, Facultad de Ciencias Naturales e Instituto Miguel Lillo, Universidad Nacional de Tucumán. San Miguel de Tucumán.

Methfessel C. y Methfessel L. 1998. Cúpulas en Rocas de Tarija y Regiones Vecinas. Primera Aproximación. Boletín 12: 36-47. SIARB, La Paz.

Nardi, R. y Chertudi, S. 1969-1970 "Instrumentos arcaicos para majar y moler en San Juan (Argentina)". Revista de Etnografía nro. 26 y 27. Volumne XII Tomo 2 Pp: 387-418. Volumen XIV, Tomo $1 \mathrm{Pp}$ : 137-188. Museu de Etnografía e Historia. Junta Distrital Do Porto, Brasil.

Querejazu Lewis, R. 1998. Tradiciones de Cúpulas en el Departemento de Cochabamba. Boletín 12: 48-58. SIARB, La Paz.

Reynoso, A. 2003. Arqueoastronomía en Rincón Chico (Catamarca, Argentina). Monumentos del tiempo, monumentos de encuentro en el valle de Yocavil. Editado por P. Cornell y P. Stenborg. Local, Regional, Global: prehistoria, protohistoria e historia en los Valles Calchaquíes. Anales Nueva Época 6:127-162. Instituto Iberoamericano, Gotemburgo.

Rusconi, C. 1945 "Molinos y morteros indígenas de Mendoza". Anales de la Sociedad Científica Argentina. Entrega III, Tomo CXXXIX. Pp. 99-119. Buenos Aires.

Scattolin, M. C., Bugliani, M. F., Cortés, L. I., Calo, C. M., Pereyra Domingorena, L., Izeta, A. D. 2009. Pequeños mundos: hábitat, maneras de hacer y afinidades en las aldeas del valle del Cajón, Catamarca. Relaciones de la Sociedad Argentina de Antropología 34:249-272. 
Serrano, A. 1945 "Los Comechingones". Imprenta de la Universidad Nacional de Córdoba. Córdoba.

Tarragó, M. 1987. Sociedad y sistema de asentamiento en Yocavil. Cuadernos del INAPL 12: 179-196.

Tarragó, M. 1998. El patrimonio arqueológico del Valle de Santa María en peligro: El Rincón Chico. En Homenaje a Alberto Rex González, 50 años de aportes al desarrollo y consolidación de la Antropología Argentina, pp.205-254, FFyL, UBA. Buenos Aires.

Tarragó, M. 2011 Poblados tipo pukara en Yocavil. El plano de Rincón Chico 1, Catamarca, Argentina. 11: 3361, Instituto Interdisciplinario Tilcara, FFyL, UBA.

Tarragó, M., L. R. González, P. Corvalán, R. Doro, M. Manasiewicz y J. Peña 1999. La producción especializada de alimentos en el asentamiento prehispánico tardío de Rincón Chico, provincia de Catamarca. Cuadernos del INAPL 18: 409-427.

van Hoek, M. 2003 "Tacitas or cupules? An attempt at distinguishing cultural depressions at two rock art sites near Ovalle, Chile". Rupestreweb, http://rupestreweb. tripod.com/tacitas.html 